九州大学学術情報リポジトリ

Kyushu University Institutional Repository

\title{
Acceleration of EC Convergence with Landscape Visualization and Human Intervention
}

Hayashida, Norimasa

Kyushu Institute of Design

Takagi, Hideyuki

Kyushu Institute of Design : Associate Professor

ht tp://hdl. hand le. net/2324/1670055

出版情報: Applied Soft Computing. 1 (4)，pp.245-256，2002-05. Elsevier Science バージョン：

権利関係 : 


\title{
Acceleration of EC Convergence with Landscape Visualization and Human Intervention
}

\author{
Norimasa Hayashida Hideyuki Takagi \\ Kyushu Institute of Design \\ Shiobaru, Minami-ku, Fukuoka, 815-8540 Japan \\ Tel\&Fax: +81-92-553-4555, takagi@kyushu-id.ac.jp, http://www.kyushu-id.ac.jp/ takagi
}

\begin{abstract}
We propose Visualized EC/IEC as an evolutionary computation (EC) and interactive EC (IEC) with visualizing individuals in a multidimensional searching space in a 2-D space. This visualization helps us envision the landscape of an $n$-D searching space, so that it is easier for us to join an EC search by indicating the possible global optimum estimated in the 2-D mapped space. We first compare four mapping methods from the points of view of computational time, convergence speed, and visual easiness to grasp whole EC landscape with 5 benchmark functions and 28 subjects. Then, we choose self-organizing maps for the projection of individuals onto a 2-D space and experimentally evaluate the effect of visualization using a benchmark function. The experimental result shows that the convergence speed of GA with human search on the visualized space is at least five times faster than a conventional GA.

keywords: interactive evolutionary computation, multidimensional data visualization, human intervention, accelerating EC convergence, human fatigue, self-organizing maps
\end{abstract}

\section{Introduction}

Interactive evolutionary computation (IEC) has been used for several application tasks, for example, graphic arts and animation, 3-D CG lighting, music, editorial design, industrial design, facial image generation, speech processing and synthesis, hearing aid fitting, virtual reality, media database retrieval, data mining, image processing, control and robotics, food industry, geophysics, education, entertainment, social system [16, 17, 21]. In spite of the increasing interest, this technology still has a common problem with these applications: the fatigue of IEC users. To make this IEC technology practical, we must improve the user interface.

In a conventional IEC, the roles of EC and a human user are completely separated; the EC performs the searches while the human user evaluates the result. The user plays a passive role in the search which contributes to the slow convergence and human fatigue.

We have proposed methods that allows an IEC user to directly participate in EC searches to ease psychological and physical fatigue $[18,20,5,3]$. The active user intervention leads to a faster convergence of the EC search, and the faster convergence results in less user fatigue. On-line knowledge embedding is one based on this idea. This method provides a mechanism to accept the searching idea, hints, or intentions of an IEC user during the IEC operation [4, 18]. For example, when a user feels that a certain part image of a montage face is acceptable, we fix the partial face image in subsequent searches, which limits the searching space and, therefore, converges faster. The effect of this method was shown through subjective tests [18]. Directly editing the tree of genetic programming for $\mathrm{CG}$ is another method of active user intervention to EC search [22].

Our proposed Visualized IEC and/or Visualized EC is another method based on the same idea of active user intervention. The Visualized EC/IEC allows an EC or IEC user to actively participate in EC searches by providing the user the distribution image of past individuals mapped from an $n$-D searching 
space to a $2-\mathrm{D}$ space $[20,5,3]$.

As the user intervention relatively takes longer time in comparison with EC operations, the Visualized EC is applicable only when fitness calculation takes longer time than the user intervention. There are many such EC tasks, for example, it takes a half to two hours for some of geophysical simulations to calculate all fitness values in each EC generation $[2,3]$. On the other hand, the Visualized IEC is always applicable because the human evaluation of individuals takes long time. So that, we mainly focus on the Visualized IEC rather than Visualized EC in the following sections except experimental evaluation in section 4.

We first propose the Visualized IEC in section 2, evaluate four mapping methods for visualization with 5 benchmark function and 28 subjects in section 3, and evaluate its convergence performance using a benchmark function in section 4 . Although the main objective of this paper is to propose the Visualized $\mathrm{EC} / \mathrm{IEC}$ and evaluate the performance of its visualization, we show some its applications in section 5 to show its applicable potential. As genetic algorithm (GA) is used in our experiments as one of EC techniques, we sometimes use the term of Visualized GA/IGA in this paper.

\section{Visualized IEC}

\subsection{Why Visualized IEC ?}

Visualized IEC is a method that combines the different capabilities of EC and humans to search for a global optimum. The EC directly and systematically searches the original $n$-D space using EC operators, which is much better than the human searching capability. However, humans have an excellent capacity to grasp an entire distribution of individuals in the 2-D space at a macroscopic level that cannot be interpreted by the EC. This is why the Visualized IEC combines the algorithmic EC search in an $n$-D space and the human global search in a mapped 2-D space.

Since a human IEC user and the EC cooperate with each other and optimize their own searching advantages in the Visualized IEC, we can expect a faster convergence. This searching cooperation feature is different from a conventional IEC or previously proposed visualization-based searches. The roles of the EC and the human are separated in conventional IEC; the EC performs the search and an IEC user evaluates the searched individuals, independently. For example, the visualization-based searches used in a 3-D CG design support system [10] and a violin sound estimator [9] request human users to both search for the global optimum on the mapped 2-D searching space without the help of optimization algorithms and evaluate the searched individuals.

\subsection{Multidimensional Data Projec- tion}

Since it is difficult for humans to grasp the geometric relationship among individuals that have multidimensional parameters, it is difficult to directly obtain information from an $n$-D searching space. However, it becomes easier for humans to grasp the approximate relationships among individuals by mapping the individuals from the $n$-D searching space to a $2-\mathrm{D}$ space. Although the 2-D space does not keep all the information about the original $n$-D space, humans can grasp the state of the whole of the searching space when the topological relationships among individuals in the 2$\mathrm{D}$ space are about the same as those in the original $n$-D space, which helps humans to cooperate with the EC on search and indicate the direction to the global optimum. The indication of the search is based on the geometric relationships kept which shows that individuals with high fitness values tend to concentrate on the same points in the 2-D space (see Figure 1.)

There are several mapping methods for 2-D visualization, for example, the principle component analysis, Sammon's non-linear mapping (NLM) [15], selforganizing maps (SOM) [6], VISOR [7], TOPAS [8], and the method using genetic programming [23]. Any visualization methods are usable in the Visualized IEC, but some methods are more suitable for the Visualized IEC than others. We evaluate the differences in section 3 . 


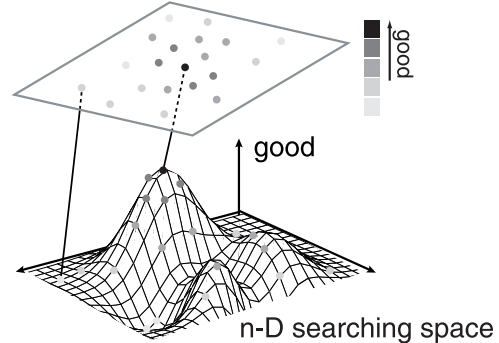

Figure 1: Projection image from an $n$-D space to a 2-D space while keeping the topological relationships among data samples.

\subsection{Construction of a Visualized IEC system}

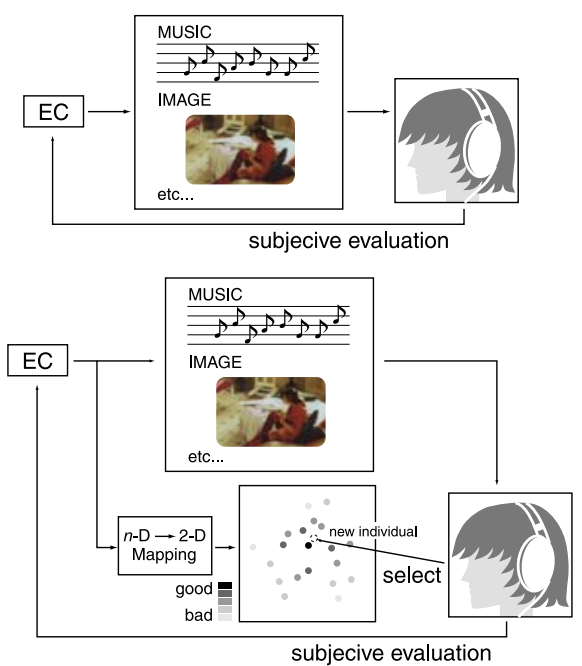

Figure 2: Diagrams of IEC (upper) and Visualized IEC (lower).

In the IEC, EC optimizes the parameters of an application task, and the task system outputs sound, speech, graphics, or other outputs which are handled by the human senses. The human IEC user observes the output, evaluates them based on his or her sense of value, and returns his or her subjective fitness value to the EC (see Figure 2 upper.)
Visualized IEC systems display not only sound or graphics to the user, but also the distribution image of past EC searched individuals by mapping them from an $n$-D searching space to a $2-\mathrm{D}$ space (see Figure 2 lower.) Fitness values of individuals in the 2-D space are displayed with gray level, different colors, the depth of color, size, or numerical characters to view, for example, to visualize the landscape shape of a searching space. An IEC user selects points in the 2-D space that look to have high fitness values from the fitness value distribution point of view, and the individual with the lowest fitness value in the EC population is replaced with the selected best point. Since neighboring individuals in an $n$-D space becomes neighbors in a 2-D space too, the topological relation of the $n$-D space is kept in the mapped 2-D space and the additional selected individual in the 2D space can be expected to be located near the global optimum in the $n$-D space. The EC mates and creates offspring using the parent population that includes the newly added individual. See these operation flow in Figure 3.

Since the possibility that an excellent individual is added to the EC population in each generation by the IEC user is high, the acceleration of EC convergence is expected.

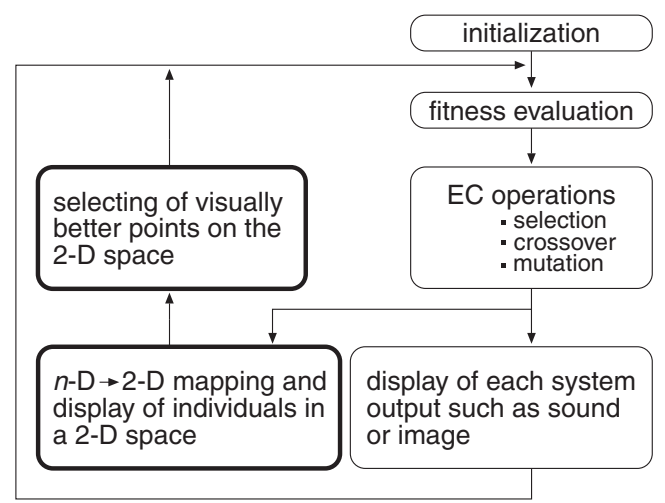

Figure 3: Flow of Visualized EC/IEC. Process in bold boxes are for visualization. Fitness evaluation is conducted by a fitness function or human subjective evaluation in Visualized EC and Visualized IEC, respectively. 


\section{Mapping Methods for Visu- alized EC/IEC}

We evaluate four mapping methods from the view point of the practical use of the Visualized EC/IEC. The main technique of the Visualized EC/IEC is a mapping method from $n$-D space to 2 -D space. We should choose the methods that the global optimum must be easily estimated visually on the mapped 2-D space and whose computational cost must be in practical level. Although there are several mathematical methods that project data from $n$-D space to $2-\mathrm{D}$ space, suitability for the Visualized EC/IEC may be different.

We compare and evaluate the mapping performance and computational cost of NLM [15], VISOR [7], SOM [6], and TOPAS [8] with five benchmark functions in Table 1 in this section. The mapping method recommeneded in this section will be used in section 4 , where we evaluate the effect of the visualization.

Table 1: Five test functions. conducted with Pentium II whose clock is $400 \mathrm{MHz}$ under Linux OS.

Table 2: CPU time (in seconds) of three mapping methods for five test functions.

\begin{tabular}{|c|c|c|c|}
\hline & SOM & NLM & VISOR \\
\hline DeJong F1 & 3 & 1376 & 1 \\
\hline DeJong F3 & 4 & 1342 & 1 \\
\hline DeJong F5 & 3 & 6119 & 1 \\
\hline Schaffer F1 & 4 & 1328 & 1 \\
\hline Schaffer F2 & 4 & 2708 & 1 \\
\hline
\end{tabular}

Table 3: CPU time (in seconds) of TOPAS for Schaffer F2.

\begin{tabular}{|c|c||c|c|}
\hline \# of data & CPU time & \# of data & CPU time \\
\hline 100 & 139 & 220 & 2784 \\
\hline 120 & 237 & 240 & 3941 \\
\hline 140 & 487 & 260 & 6263 \\
\hline 160 & 813 & 280 & 8379 \\
\hline 180 & 1279 & 300 & 10870 \\
\hline 200 & 1921 & & \\
\hline \multicolumn{4}{|l}{} \\
\hline
\end{tabular}

The TOPAS did not stand comparison with these three methods; it is too slow. We gradually increased the number of data, calculated the CPU time with $\$ c h a f f e r ' s$ second function, and obtained the data in Table 3. The approximation function for these CPU time data was $t=10^{-6} * n^{3.9851}$. Its complexity seems $O\left(n^{4}\right)$, which is supported by the fact that the TOPAS program includes quadruple loops. This complexity means that the TOPAS needs 452 days to map 2,500 data.

These experimental results regarding computational cost conclude that (1) the NLM and the TOPAS are far from practical use for Visualized EC and Visualized IEC, and (2) the VISOR is the best, but the SOM is also within permissible range of practical use. We adopt only the VISOR and SOM in the following two experiments. 


\subsection{Comparison by Visual Inspection}

Mapping result to a 2-D space must be easy for a human user to visually estimate the global optimum. We evaluate the visual impression of the mapped images of the SOM and VISOR using human subjects because NLM and TOPAS are far from practical use shown in section 3.1. We request 28 students in twenties to operate the Visualized GA with SOM and the Visualized GA with VISOR for the 5 benchmark functions in Table 1 and report which Visualized GA is easier to grasp whole landscape of GA search space and easier to visually find better individuals. Their comparisons are statistically tested using a sign test. See the result in Table 4.

Table 4: Sign test results of 28 subjects on visual inspection of two Visualized GA. SOM and VISOR mean that the number of the case that Visualized GA with SOM or VISOR were easier to guess where are better points on the 2-D map than another; SAME means that visual inspection of two Visualized GA were same; ${ }^{* *}$ means significance with $(p<0.01)$.

\begin{tabular}{|c|r|r|r|c|}
\hline $\begin{array}{c}\text { benchmark } \\
\text { function }\end{array}$ & SOM & VISOR & SAME & $\begin{array}{c}\text { sign } \\
\text { test }\end{array}$ \\
\hline DeJong F1 & 27 & 0 & 1 & $* *$ \\
\hline DeJong F3 & 23 & 2 & 3 & $* *$ \\
\hline DeJong F5 & 22 & 1 & 5 & $* *$ \\
\hline Schaffer F1 & 25 & 2 & 1 & $* *$ \\
\hline Schaffer F2 & 25 & 0 & 3 & $* *$ \\
\hline
\end{tabular}

Figure 4 is example visualizations of GA landscape at the fifth generation when the Visualized GA with SOM and the Visualized GA with VISOR are applied to Schaffer F2. The mapping relation between $n$-D and 2-D spaces are previously calculated using 2,500 data which corresponds to resolution of the map. Fitness value at each searching point is expressed by the depth of color in reality.

Experimental result has shown than the visualization by SOM seems to have more visual information of GA landscape than that by VISOR. Searching points are widely spread by SOM in the Figure 4, while those by VISOR locally gather in some spots.

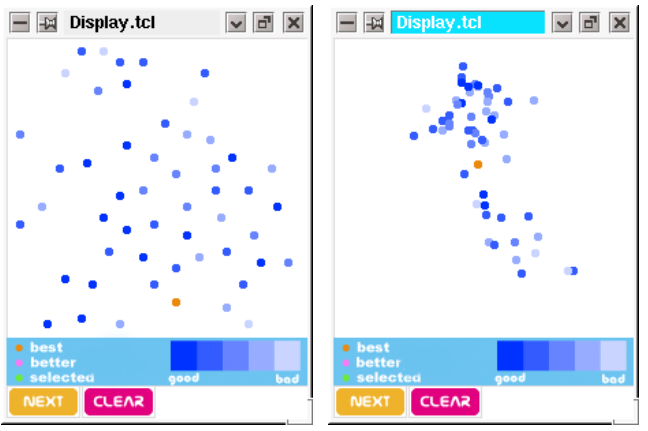

Figure 4: Example visualizations of GA landscape: the Visualized GA with SOM (left) and the Visualized GA with VISOR (right).

This implies that the VISOR visually provides less information of the GA landscape than the SOM does. In fact, many subjects reported that the visualization by SOM was easier to guess where are better points on the 2-D map than that by VISOR.

\subsection{Comparison of Convergence of two Visualized GAs}

The convergence of the Visualized GA with SOM and the Visualized GA with VISOR is compared using five benchmark functions in Table 1. Same GA conditions are used for all experiments; GA coding type is 32 bits real coding, crossover rate is 0.9 , mutation rate is 0.02 , and population size is 20 . Mapping relationships from $n$-D to $2-\mathrm{D}$ using SOM and VISOR were previously calculated with 10,000 data for visualization GAs. The average convergence curves are calculated using the data of 28 subjects.

Figure 5 shows average curves of 28 subjects for two Visualized GAs. Subjective tests with Visualized GAs were conducted till the fifth generations because the convergence of the Visualized GAs became clear at the fifth generation and it is important to keep subjects with less fatigue for reliable subjective tests.

The convergence of two Visualized GA at the fifth generation are evaluated using a sign test. Table 5 shows the test results. The reason why Figure 5(c) seems different from the result in Table 5 is that the 

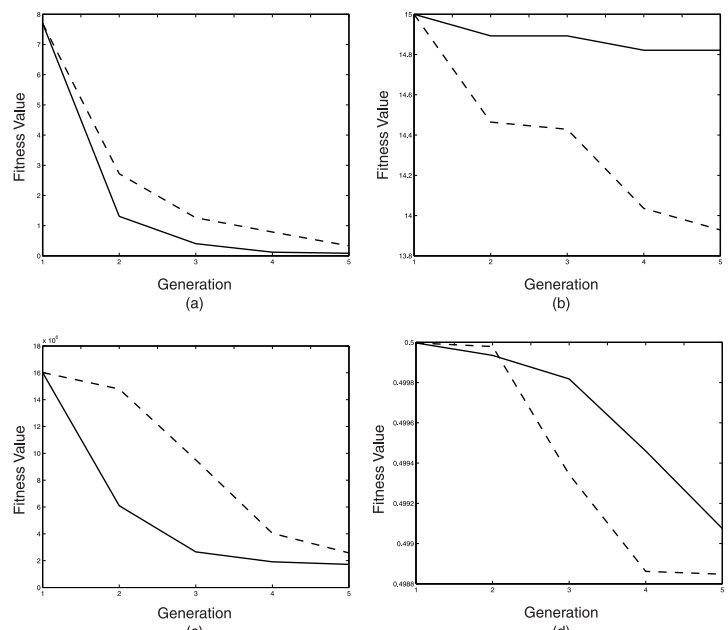

(c)

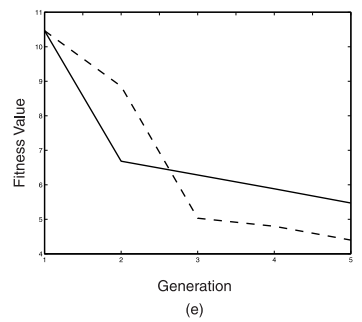

- - - Visualized GA with VISOR — Visualized GA with SOM

Figure 5: Average convergence curves of two Visualized GAs for (a) DeJong's F1, (b) DeJong's F3, (c) DeJong's F5, (d) Schaffer's F1, and (e) Schaffer's F2.

Table 5: Sign test results of 28 subjects on convergence of two Visualized GA. SOM and VISOR mean that the number of the case that Visualized GA with SOM or VISOR were faster than another; SAME means that convergence of two Visualized GA were same; $* *$ and $*$ means significance with $(p<0.01)$ and $(p<0.05)$

\begin{tabular}{|c|r|r|r|c|}
\hline $\begin{array}{c}\text { benchmark } \\
\text { function }\end{array}$ & SOM & VISOR & SAME & $\begin{array}{c}\text { sign } \\
\text { test }\end{array}$ \\
\hline DeJong F1 & 23 & 5 & 0 & $* *$ \\
\hline DeJong F3 & 3 & 7 & 18 & \\
\hline DeJong F5 & 20 & 8 & 0 & $*$ \\
\hline Schaffer F1 & 20 & 8 & 0 & $*$ \\
\hline Schaffer F2 & 8 & 20 & 0 & $*$ \\
\hline
\end{tabular}

average curves are deeply influenced by extremely better/poor convergence curve among 28 subjects, while sign test does not consider the amount of difference between two curves. The SAME for DeJong F3 means that 18 subjects could not converge and within first five generations.

These results imply that the superiority of two Visualized GA depends on tasks.

\subsection{Discussion}

The summary of three experimental results in this section are: (1) the VISOR needs less calculation time than the SOM, (2) the visualization by SOM is easier to visually grasp whole GA landscape than that by VISOR, and (3) superiority of two mapping methods in convergence depends on tasks.

Visualized EC is applicable only when fitness calculation takes longer time than human interaction on the visualized 2-D map, while Visualized IEC is always applicable as mentioned before. From this applicable point of view, we decide to attach importance to the human aspect of two mapping methods, i.e. we consider that SOM is more important to reduce the fatigue of Visualized IEC users. We use only SOM for the visualization in the subjective experiment in the next section. Note that the reason why we adopt Visualized GA in the next section is for objective evaluation of the Visualized IGA, and it does not contradict our attaching importance to the SOM for Visualized IEC.

\section{Evaluation of Convergence}

\subsection{Experimental System}

We evaluate how human intervention accelerates the convergence of the EC search. The final evaluation should be conducted using the Visualized IEC and subjective tests. However, since the IEC deals with subjective fitness values that depend on the application task and the subject's perceived value of the task, we preliminarily evaluate the effect of the human intervention without subjective evaluation to the given tasks in this section. 


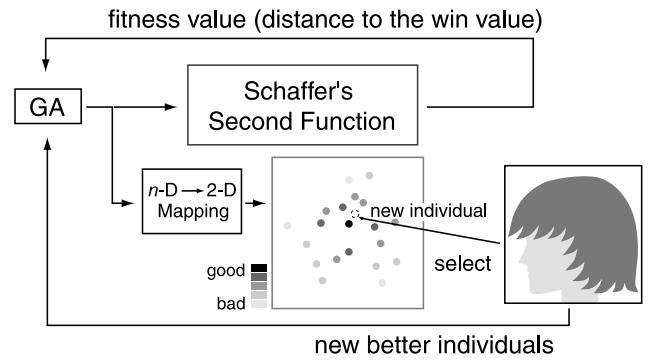

Figure 6: Experimental system of the Visualized GA. GA determines the coordinate of the minimum value of the Schaffer's second function, and the difference of the function output and the minimum value is fedback into the GA as a fitness value. The human operator visually selects a possible global optimum in the mapped 2-D space and sends it to the GA as a new possible parent. Self-organizing map is used to map individuals from an $n$-D space to a 2-D space.

We do not compare the convergence performance of the Visualized IEC and IEC but that of the Visualized EC and EC in reality; since we use GA as one of the EC technologies in this section, the actual comparison is the Visualized GA versus a normal GA. This experimental evaluation needs no human interactive evaluation of the given task but only the human selection of better individuals in the 2-D space.

Figure 6 shows an experimental system. Since this system has a fitness function, the role of the human user is only to select better candidates in the 2-D visualized space, while a Visualized IEC user plays both roles of a fitness function and a selector of the better candidates (compare Figures 2 and 6.)

Two modified Schaffer's second functions illustrated in Figure 7 in 3-D and 5-D are used as the experimental tasks of the Visualized GA and GA. Flat space at the bottom in Figure 7 is a searching space, and the shape of the function is a landscape of the searching space. The searching points on the landscape are mapped onto 2-D space, and their fitness values are displayed. The task is to determine the coordinate that results in the minimum value of the Schaffer's function, i.e. 0. Distance, |function (GA individual) $-0 \mid$, is fed-back into the GA as a fitness value.

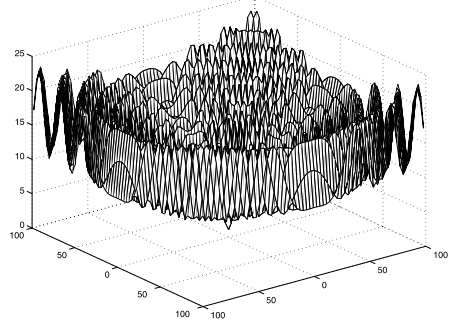

Figure 7: Modified Schaffer's second function given by $\left(\sum_{i=1}^{n} x_{i}^{2}\right)^{\frac{1}{4}} *\left\{\sin ^{2}\left(50 *\left(\sum_{i=1}^{n} x_{i}^{2}\right)^{\frac{1}{10}}+1\right\}\right.$, where $-100 \leq x_{i} \leq 100$ and $n=3$ and 5 .

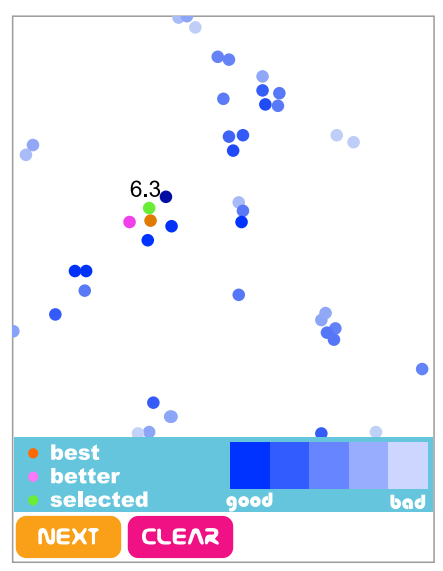

Figure 8: The example image of the mapped 2-D space of 40 individuals $(=20$ individuals $\times 2$ generations) in the second generation and some individuals created by a user. Original interface distinguishes individuals with color.

Figure 8 is the interface design of the mapped 2D space used in our experiment. The fitness values of individuals, including those from past generations, are displayed with different depths of color. A human user finds an area where individuals with higher fitness values gather, selects a maximum of three individuals that seem to have the highest fitness values, and replaces one of the 20 individuals with the se- 
lected one. Besides the classification by the five levels of depth of blue color, our experimental system can display the exact fitness value of the selected point so it is easier for users to compare individuals. The best individual in each generation is displayed with an orange color, and if the fitness value of a new individual created by the user is better than these best individuals, the new one is displayed in pink.

Table 6: Experimental conditions of GA and SOM.

(a) GA parameters

\begin{tabular}{|c|c|}
\hline population size & 20 \\
crossover rate & 0.9 \\
mutation rate & $1 / 80$ \\
\# of generations & 10 \\
GA coding & binary coding \\
bit length & 16 \\
\hline
\end{tabular}

(b) SOM parameters

\begin{tabular}{|c|c|}
\hline \# of learning & 1,000 \\
neighborhood function & step function \\
shape of neighborhood & hexagon \\
neighborhood radius & 5 \\
learning rate factor & 0.4 \\
\# of units & $200 \times 200$ \\
\hline
\end{tabular}

We adopt SOM to map individuals from an $n$-D space to a 2-D space as seen in Figure 1 from the discussion in section 3 . Figure 8 is the example of mapped distribution of individuals. The population size is 20 . As the amount of data increases, we retrain the SOM in every generation, so that the number of displayed individuals in the $2-\mathrm{D}$ space increases 20 by 20 in each generation. The possible mapped points in the $2-\mathrm{D}$ space are $200 \times 200$.

SOM learns all mapping points on a $2-\mathrm{D}$ space from input data in an $n$-D space before it is used in the $\mathrm{Vi}$ sualized EC/IEC. Therefore, it is quite easy to find the point in the $n$-D space that corresponds to the user's choice on the 2-D space during searching process of the Visualized EC/IEC. Since the SOM does not keep the absolute distance relationship in the $n$ $\mathrm{D}$ space on the $2-\mathrm{D}$ space, we cannot estimate the distances among points in the $n$-D space from those in the 2-D space. However, the SOM keeps topological relationship of these distances, and we can expect that the similar evaluation would be given to their neighbor points.

The experimental conditions of GA and SOM are showed in Table 6 . The evaluating subjects in our experiment are five graduate and undergraduate students.

\subsection{Experimental Results}

Figure 9 shows the experimental results for the tasks whose complexity is different, where the dimensional number, $n$, is 3 and 5 . These graphs clearly show that the visualized GA converges much faster than a normal GA; the convergence speed of the Visualized GA with a population size of 20 is similar to that of normal GA with a population size of 100 and 1,000. This means we can expect that the Visualized GA to converge five time faster or more than the normal GA.

\subsection{Discussion}

Although the convergence characteristics of our proposed method depends on the subjects, the average convergence characteristics of the five subjects was much faster than that of normal GA. Due to human fatigue, the number of EC generations and the number of individuals displayed to an IEC user was usually limited to 20 generations and 20 individuals at most. Therefore, the experimental result that the proposed method with a few individuals converges faster than the normal GA with many individuals implies that the Visualized IEC is expected to be a powerful tool for difficult IEC tasks.

Generally, the higher the dimensional number of a searching space, the more slowly the GA converges. In our experimental evaluation, the convergence performances of some experimental subjects did not depend on the dimensional number, 3 and 5 . There was even a case that convergence in a 5 -D space was better than that in 3-D space. We can expect that the convergence of the Visualized IEC is tolerant about 

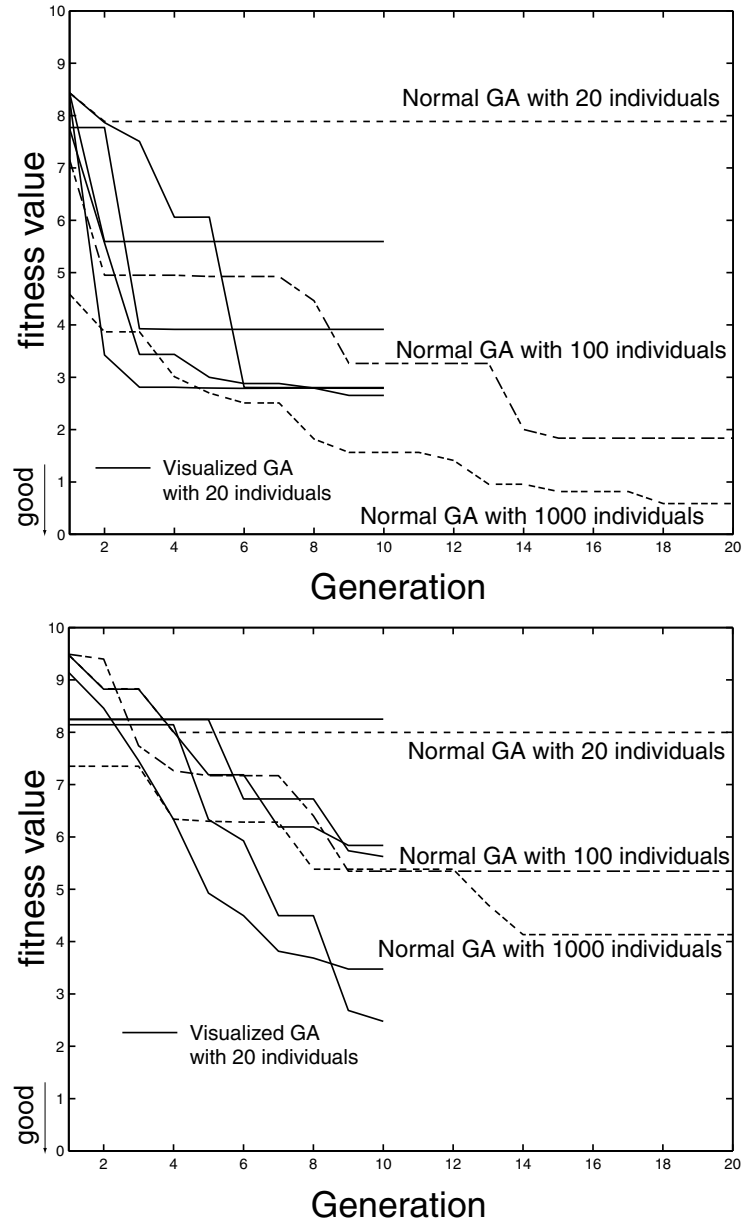

Figure 9: Convergence characteristics of normal GAs with population sizes of 20,100, and 1,000 and the Visualized GA with a population size of 20 for a modified Schaffer's function of 3-D and 5-D, are shown in the upper and lower graphs, respectively. Solid lines represent the five experimental subjects for the Visualized GA. the complexity of tasks and that the Visualized IEC becomes much more powerful for complex tasks.

In early generations, when the individuals are sparsely displayed in the $2-\mathrm{D}$ space, it is often difficult to observe the landscape of the searching space, which makes it difficult for human subjects to estimate the location of the global optimum in the 2-D space. For such a case, they choose points near the best individuals in the 2-D space. As these points are expected to have higher fitness values as well as the best individuals, the sparse distribution in the early generation may not been a serious problem.

Even if IEC users select and create a worst individual, it mean that only one bad individual is added to many in a population, and it is weeded out by the natural selection in the next generation, which is not a serious problem.

In our experiment, we found that the training time of SOM to map 40,000 points $(=200 \times 200$, see Table $6(\mathrm{~b})$ ) in every generation would be too long for a smooth human interaction with our computer used in section 3. The easiest solution is to reduce the number of SOM units. After the experiment described in this section, we confirmed that the SOM calculation time could be decreased within an allowable range without reducing its performance by reducing the number of SOM units.

\section{Applications of Visualized IEC}

The experimental evaluation in the previous section has shown the effectiveness of visualization for GA search, and we can expect the Visualized IEC is a practical solution for several applications. In this section, we show two examples of Visualized IEC systems.

\subsection{Application to Speech Processing}

Figure 10 is a user interface of the Visualized IEC applied to a speech processing system. The IEC speech processing system is a system that improves the quality of speech sound [24]. The frequency characteristics of a filter is specified by 6 parameters of the 


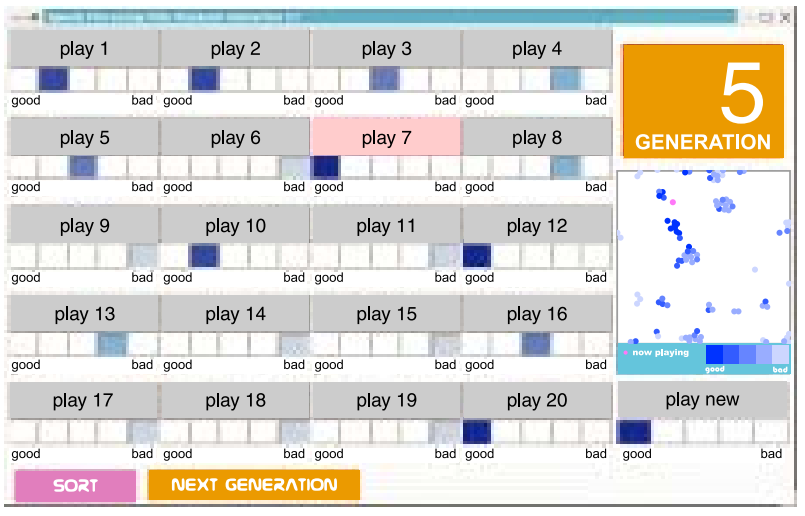

Figure 10: User interface of the Visualized IEC for speech processing.

amplification levels at $125 \mathrm{~Hz}, 250 \mathrm{~Hz}, 500 \mathrm{~Hz}, 1 \mathrm{kHz}$, $2 \mathrm{kHz}$, and $4 \mathrm{kHz}$. These parameters are modified to improve the speech quality based on user's hearing and GA search. The processed speech sounds are presented when play buttons on the IEC user interface are pressed. An IEC user evaluates each processed sound, and the GA searches better filter parameters based on user's subjective evaluation as fitness values.

Besides the user interface of the IEC consisting of play buttons and rating buttons for 20 individuals, the interface of the Visualized IEC has a window for data visualization located on the right side of Figure 10. Six dimensional filter parameter vectors are mapped by SOM on the 2-D window and displayed at once. Darker colors are assigned to higher rating buttons, and the same depth of color on the 2-D windows indicates a similar human evaluation of filters in past generations. A Visualized IEC user clicks points where he or she guesses that there are better parameter vectors of filters from the distribution of rated past ones on the 2-D space. Then, a new filter is created from the location information of the clicked point, and the speech sound processed by the filter is displayed to the user. The user evaluates the filter in the same way. The user can repeat to create a better filter until he or she is satisfied, and final created best filter is used as a new parent when the next generation button at the bottom of the figure is pushed.

Figure 10 shows the distribution of the 5 th generation of the Visualized IEC for speech processing, and 20 individuals $\times 5$ generations and some individuals newly created by a user are displayed on the 2-D windows. Observing the distribution of fitness values in this figure, it looks like many better individuals are located within the same narrow area on the 2-D window. It seems helpful for the user to quickly find the filter that minimally distorted the speech sound by searching near that place.

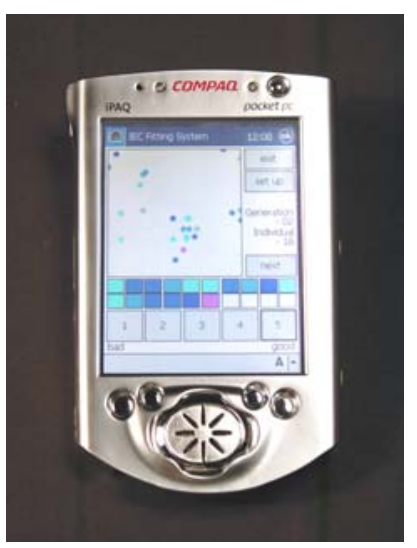

Figure 11: Graphical user interface of a PDA version of an IEC-based hearing aid fitting.

Another IEC application to speech processing is an IEC-based hearing aid fitting, IEC Fitting $[11,12$, 13, 19]. As one of the features of the IEC Fitting is fitting place-free, portability is important for the IEC Fitting to let hearing aid users and audiologists fit their hearing aids in their daily sound environment.

A PDA (Personal Digital Assistant) version of the IEC Fitting was developed for this purpose. To put all user interface on to the small display of a PDA, the Visualized IEC was use (see Figure 11). 


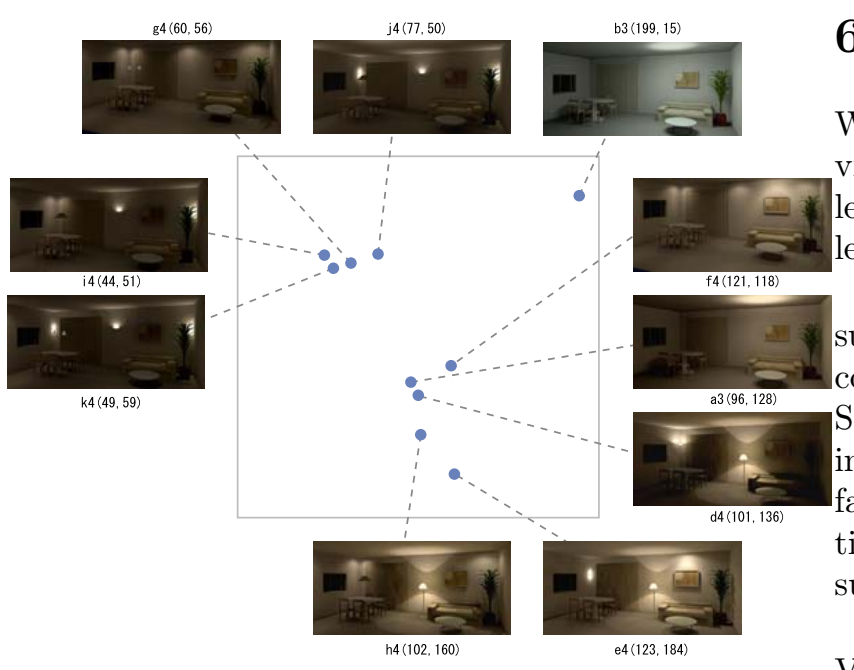

Figure 12: Distribution of room lighting parameters mapped on 2-D space and corresponding CG lighting expression for night scenes.

\subsection{Application to Room Lighting De- sign}

We are expanding our previous work of a 3-D CG lighting design support system [1] to a room lighting design. The task of 3-D CG lighting is to determine the best positions and brightness of multiple lights and create a lighting expression by matching the effect to the given motif or personal preference.

Figure 12 is the distribution of 10 room lighting parameter vectors mapped from a 7 -D parameter space to a 2-D space. Lighting CG scenes created by 10 lighting parameter vectors are posted in the same figure like as Marks et al.'s Design Galleries [10] for the readers' convenience. Since similar CG scenes tend to gather in the similar location in the $2-\mathrm{D}$ space, it expected that the users could easily and quickly find an ideal lighting for them.

We are going to evaluate the convergence characteristics of the Visualized IEC approach by comparing it with a purely manual searching approach like the Design Galleries [10] or an acoustic data navigator $[9]$.

\section{Conclusion}

We proposed the Visualized IEC that provides the visual landscape of searching space to IEC users and lets them actively participate in EC searching with less fatigue, which results in a faster EC convergence.

We first compare four mapping methods for the visualization and choose SOM. Then, we evaluated the convergence performance of the Visualized GA with SOM and normal GA. The experimental evaluation implied that the Visualized IEC converges five times faster or more than a normal IEC, though this quantitative performance value depends on GA condition, subjects, and tasks.

We then showed two example applications of the Visualized IEC, observed the distribution of data in the mapped 2-D space, and found that the individuals whose phenotypes are similar gather and become neighbors in the mapped 2-D space. We are going to continue further evaluation whether a good individual, i.e. a solution of these concrete application tasks, can be found quickly through subjective tests.

\section{References}

[1] Aoki, K. and Takagi, H.: "3-D CG lighting with an interactive GA," 1st Int'l Conf. on Conventional and Knowledge-based Intelligent Electronic Systems (KES'97), Adelaide, Australia, pp.296-301 (May, 1997).

[2] Boschetti, F. and Moresi, L.: "Comparison between interactive (subjective) and traditional (numerical) inversion by genetic algorithms," Congress on Evolutionary Computation (CEC2000), La Jolla, CA, USA, pp.522-528 (July, 2000).

[3] Boschetti, F. and Takagi, H.: "Visualization of EC Landscape to Accelerate EC Conversion and Evaluation of its Effect," Congress on Evolutionary Computation (CEC2001), Seoul, Korea, pp.880-886 (May, 2001).

[4] Caldwell, C. and Johnston, V. S.: "Tracking a criminal suspect through "face-space" with a genetic algorithm," 4th Int'l Conf. on Genetic Algorithm (ICGA'91), San Diego, CA, US, Morgan Kaufmann Publisher, pp.416-421 (July, 1991). 
[5] Hayashida, N. and Takagi, H.: "Visualized IEC: Interactive Evolutionary Computation with Multidimensional Data Visualization," IEEE Int'l Conf. on Industrial Electronics, Control and Instrumentation (IECON2000), Nagoya, Japan, pp.2738-2743 (Oct., 2000).

[6] Kohonen, T.: Self-Organizing Maps, Springer-Velag, Heidelberg (1995).

[7] König, A., Bulmahn, O., and Glesner, M.: "Systematic methods for multivariate data visualization and numerical assessment of class separability and overlap in automated visual industrial quality control," 5th British Machine Vision Conf., vol.1, pp.195-204 (Sept., 1994).

[8] König, A.: "A survey of multivariate data projection, visualization and interactive analysis," 5th Int'l Conf. on Soft Computing and Information/Intelligent Systems (IIZUKA'98), Iizuka, Japan: World Scientific, Singapore, pp.55-59 (Oct., 1998).

[9] König, A., Blutner, F. E., Eberhardt, M., and Wenzel, R.: "An acoustic data base navigator for the interactive analysis of psycho-acoustic sound archive," 5th Int'l Conf. on Soft Computing and Information/Intelligent Systems (IIZUKA'98), Iizuka, Japan: World Scientific, Singapore, pp.60-63 (Oct., 1998).

[10] Marks, J., Andalman, B., Beardsley, P. A., et al: "Design Galleries: A general approach to setting parameters for computer graphics and animation," 24th Int'l Conf. on Computer Graphics and Interactive Techniques (SIGGRAPH'97), pp.389-400 (Aug., 1997).

[11] Ohsaki, M. and Takagi, H.: "Application of Interactive Evolutionary Computation to Optimal Tuning of Digital Hearing Aids," 5th Int'l Conf. on Soft Computing (IIZUKA'98), pp.849-852, World Scientific, Iizuka, Fukuoka, Japan, (Oct., 1998).

[12] Ohsaki, M. and Takagi, H.: "Design and Development of an IEC-based Hearing Aids Fitting System," 4th Asia Fuzzy System Symposium (AFSS'00), Tsukuba, Japan, pp.543-548 (May/June, 2000).

[13] Ohsaki, M., Sakamoto, S., and Takagi, H.: "Development and Evaluation of an IEC Fitting System for Hearing Aids," Int'l Conf. on Acoustics (ICA2001), Rome, Italy, 5A.15.02 (Sept., 2001).

[14] Pratihar, D. K., Hayashida, N., and Takagi, H.: "Comparison of Mapping Methods to Visualize the EC Landscape," 5th Int'l Conf. on Knowledge-Based Intelligent Information Engineering Systems \& Allied Technologies, Osaka, Japan, pp.223-227 (Sept., 2001).
[15] Sammon, J. W.: "A nonlinear mapping for data structure analysis," IEEE Trans. on Computers C-18, no.5, pp.401-409 (1969).

[16] Takagi, H.: "Interactive Evolutionary Computation: System Optimization Based on Human Subjective Evaluation," IEEE Int'l Conf. on Intelligent Engineering Systems (INES'98), Vienna, Austria, pp.1-6 (Sept., 1998).

[17] Takagi, H.: "Interactive Evolutionary Computation - Cooperation of computational intelligence and human KANSEI -," 5th Int'l Conf. on Soft Computing and Information/Intelligent Systems (IIZUKA'98), Iizuka, Fukuoka, Japan: World Scientific, Singapore, pp.41-50 (Oct., 1998).

[18] Takagi, H. and Kishi, K.: "On-line knowledge embedding for an interactive EC-based montage system," 3rd Int'l Conf. on Knowledge-Based Intelligent Information Engineering Systems, Adelaide, Australia, pp.280-283 (Aug./Sept.,1999).

[19] Takagi, H. and Ohsaki, M.: "IEC-based Hearing Aids Fitting," IEEE Int'l Conf. on System, Man, and Cybernetics (SMC'99), Tokyo, Japan, vol.III, pp.657662 (Oct., 1999).

[20] Takagi, H.: "Active User Intervention in an EC Search," Int'l Conf. on Information Sciences (JCIS2000,) Atlantic City, NJ, USA, pp.995-998 (Feb./Mar., 2000).

[21] Takagi, H.: "Interactive Evolutionary Computation: Fusion of the Capacities of EC Optimization and $\mathrm{Hu}-$ man Evaluation," Proceedings of the IEEE, Vol. 89, No. 9, pp. 1275-1296 (Sept., 2001).

[22] Unemi, T.: "SBART 2.4: breeding 2D CG images and movies and creating a type of collage," 3rd Int'l Conf. on Knowledge-Based Intelligent Information Engineering Systems, Adelaide, Australia, pp.288-291 (Aug./Sept.,1999).

[23] Venturini, G., Slimane, M., Morin, F., and Asselin de Beauville, J. P.: "On using interactive genetic algorithms for knowledge discovery in databases," 7th Int'l Conf. on Genetic Algorithms, Morgan Kaufmann Publisher, pp.696-703 (1997).

[24] Watanabe, T. and Takagi, H.: "Recovering system of the distorted speech using interactive genetic algorithms," IEEE Int'l Conf. on Systems, Man and Cybernetics, Vancouver, Canada, vol.1, pp.684-689 (Oct., 1995). 